\title{
Remarks on the Analyticity of the Free Boundary for the One-Dimensional Stefan Problem $\left(^{*}\right)(* *)$.
}

\author{
Lev Rubinstein (Jerusalem) \\ antonto Fasano (Firenze) - Mario Primicerio (Firenze)
}

Sunto. - Si dimostra che nel problema di Stefan (con una o due fasi) nelle variabili $x \in(-\infty,+\infty)$
e $t \geqslant 0$ il contorno libero è una funzione analitica di $t^{\frac{1}{2}}$ per $t \geqslant 0$, se il dato iniziale è assunto
in una classe di funzioni intere. I coefficienti dello sviluppo del contorno libero in serie di
potenze di $t^{\frac{1}{2}}$ sono determinati mediante formule ricorrenti. Un particolare esame è svolto per
il caso in cui non c'è raccordo tra il dato iniziale e quello sul contorno libero, dimostrando
l'esistenza di soluzioni dotate di contorno analitico, sotto opportune limitazioni sul salto tra
$i$ due dati; sono però messi in evidenza anche casi in cui il problema non ammette soluzioni.
Infine una breve analisi è svolta per $i$ problemi in domini limitati, fornendo una dimostra-
zione della analiticità del contorno libero rispetto a $t$ per $t>0$, alternativa a quella di $[1]$.

\section{1. - Introduction.}

Let us consider the following one-dimensional, one-phase Stefan problem

$$
\begin{aligned}
& u_{x x}-u_{t}=0, \quad-\infty<x<y(t), \quad t>0, \\
& u(x, 0)=f(x), \quad-\infty<x<0, \\
& u(y(t), t)=0, \quad t>0, \\
& \dot{y}(t)=u_{x}(y(t), t)-\varphi(t), \quad t>0, \\
& y(0)=0,
\end{aligned}
$$

where $f$ and $\varphi$ are given functions (standing for the initial temperature and the ablation term, when (1.1)-(1.5) represents a diffusion process with change of phase) (1).

From the results of [1] it can be deduced that if $\varphi(t)$ is analytic in $\left(0, t_{0}\right)$ for some $t_{0}>0$, then any solution of $(1.1)-(1.5)$ existing in $\left(0, t_{1}\right)$ is such that $y(t)$ is analytic in the open interval $(0, \bar{t}), \bar{t}=\min \left(t_{0}, t_{1}\right)$.

(*) Entrata in Redazione il 30 maggio 1979.

(**) Work partially supported by the Italian CNR, while the first of the Authors was visiting the Mathematical Institute "U. Dini " of the University of Florence.

(1) Two-phase problems will be considered in Section 4. 
On the other hand, finding polynomial approximations of the free boundary in terms of the initial datum is a problem of obvious interest for practical purposes. Thus, a natural question arises about the analyticity of $y(t)$ at $t=0$.

However, this case occurs only under very special assumptions on $f(x)$ : it is easily seen (Remark 2) that the analyticity of $f(x)$ is a necessary condition which is far from being sufficient; necessary and sufficient conditions will be found in Section 3 (Remark 3) as a consequence of the main result of the paper, namely:

THEonem 1. - Assume that $\varphi$ is analytio w.r.t. $\sqrt{t}$ in $\left[0, t_{0}\right]$ for some $t_{0}>0$ and that $f$ is an entire function of $x$ such that

$$
\begin{gathered}
f(0)=0 \\
\left|f^{\prime}(x)\right| \leqslant M \exp \left(\alpha x^{2}\right)
\end{gathered}
$$

for some positive constants $M$ and $\alpha$. Then there exists $t_{1} \in\left(0, t_{0}\right]$ such that (1.1)-(1.5) has one unique solution and the free boundary $x=y(t)$ has the following series representation

$$
y=\sum_{n=2}^{\infty} Y_{n} t^{n / 2} / n !, \quad t \in\left[0, t_{1}\right)
$$

The proof is given in Section 2 by the use of Gevrey's method of continuation of heat potentials and of Poisson's integral into the complex plane [3] $\left({ }^{2}\right)$ : the integral equations determining $v(t)=u_{x}(y(t), t)$ and $y(t)$ are written according to Rubinstein's method [2], the variable $T=\sqrt{ } \bar{t}$ is introduced and the function $v\left(T^{2}\right)$ is extended to the complex plane $T=\varrho e^{i \varphi}$; then a circle $|T|<R_{0}$ is found in which the integral equations are solved by the fixed point of a contractive mapping of a suitable set of analytic functions into itself. At this point this analyticity property can be extended to the whole existence interval applying the usual prolongation method, or referring to the results of [1].

In Section 3 we find recursive relations, giving the coefficients of the power expansion of the free boundary w.r.t. $\sqrt{t}$ in terms of $f$ and $\varphi$ and vice-versa.

Section 4 is devoted to the study of the case $f(0) \neq 0$ and to other extensions of Theorem 1. Then main result in this section is

THEOREM 2. - Assume that $\varphi(t) \sqrt{t}$ is analytio w.r.t. $\sqrt{t}$ in $\left[0, t_{0}\right]$, that $|\varphi(t) \sqrt{t}| \leqslant \varphi_{0}$, and that $\lim _{x \rightarrow 0^{-}} f(x)=f_{0}$. Then, if the other assumptions of Theorem 1 are satisfied, two positive constants $\varphi_{0}^{*}, f_{0}^{*}$ can be found such that for any $\varphi_{0} \leqslant \varphi_{0}^{*},\left|f_{0}\right| \leqslant f_{0}^{*}$ problem $(1.1)-(1.5)$, has one unique solution in the class of solutions whose free boundary is analytic in $\left[0, t_{1}\right)$, for some $t_{1} \in\left(0, t_{0}\right]$, w.r.t. $\sqrt{t}$.

(2) This method has been used by H. GEIMAN [4] to study the analyticity of the free boundary w.r.t. a physical parameter in the problem of the crust growth on a plate imbedded into a melt [2]. 
Existence and uniqueness of solutions of (1.1)-(1.5) with the initial datum not vanishing at $x=y(0)$ and positive had not been proved in the literature. Therefore the part of Theorem 2 concerning existence when $f_{0}>0$ is a new contribution in this sense. For the sake of completeness, in the same section another uniqueness theorem is proved with no special regularity assumption on the solutions.

The case $t_{0} \neq 0$ is again considered in Section 5, where a nonexistence theorem is proved for $f_{0} \geqslant 1$.

The Stefan problem in bounded domains is finally discussed in Section 6, investigating the analyticity of the free boundary.

REMARK 1. - Assume $\varphi(t), y(t)$ are analytic in $[0, \hat{t}]$ and solve the non-characteristic Cauchy problem for (1.1) with analytic data

$$
\begin{array}{ll}
u(y(t), t)=0, & 0 \leqslant t \leqslant \hat{t}, \\
u_{x}(y(t), t)=\dot{y}(t)-\varphi(t), & 0 \leqslant t \leqslant \hat{t} .
\end{array}
$$

According to [3] its solution is analytic in $\boldsymbol{R} \times[0, \hat{t}]$ and in particular it is an entire function w.r.t. $x$. This means that if $(1.1)-(1.5)$ has a solution whose free boundary is analytic in the closed interval $[0, \hat{t}]$, it coincides, for $x \leqslant y(t)$, with the solution of (1.1), (1.6), (1.7) and thus with the solution of a characteristic Cauchy problem with an initial datum given by

$$
f(x)=\sum_{n=0}^{\infty} f_{n} \frac{x^{n}}{n !}
$$

where the coefficients $f_{n}$ are found by differentiating (1.6), (1.7) along $y(t)$ at $t=0$ and using (1.1).

Thus

$$
\begin{aligned}
& f_{0}=0, \\
& f_{1}=\dot{y}(0)-\varphi(0), \\
& f_{2}=-f_{1} \dot{y}(0), \\
& f_{3}=\ddot{y}(0)-\dot{\varphi}(0)-f_{2} \dot{y}(0), \\
& f_{4}=-f_{2} \dot{y}^{2}(0)-2 f_{3} \dot{y}(0)-f_{1} \ddot{y}(0),
\end{aligned}
$$

and so on.

Therefore, it comes out that a necessary condition for the analyticity of $y(t)$ up to $t=0$ is that $f$ is an entire function such that the even order coefficients $f_{2 n}$ $(n=1,2, \ldots)$ in the expansion (1.8) are determined by the odd order coefficients $f_{2 m-1}(m \leqslant n)$ through the recursive relations (1.9).

For instance, assuming e.g. $\varphi(t)=0$, it is immediately seen that $f(x)=x$ (and more generally any odd non zero entire function) does not generate a free boundary 
which is analytic at $t=0$ : indeed the zero level curve of the corresponding characteristic Cauchy problem is $x=0$, on which (1.4) is obviously violated.

On the other hand, the same can be said if $f$ is an entire even function, since for the corresponding characteristic Cauchy problem no level curve originates from $x=0, t=0$.

\section{2. - Proof of theorem 1.}

The solution of (1.1)-(1.3) has the integral representation

$$
u(x, t)=\int_{-\infty}^{0} f(\xi) E(x-\xi, t) d \xi+\int_{0}^{t} v(\tau) E(x-y(\tau), t-\tau) d \tau,
$$

where

$$
\begin{gathered}
E(x, t)=(4 \pi t)^{-\frac{1}{2}} \exp \left[-x^{2} / 4 t\right], \\
v(t)=u_{x}(y(t), t) .
\end{gathered}
$$

Following [2], it can be seen that (1.1)-(1.5) is equivalent to the system of integral equations

$$
\begin{gathered}
v(t)=2 \int_{-\infty}^{0} f^{\prime}(\xi) E(y(t)-\xi, t) d \xi- \\
-\int_{0}^{t} v(\tau)(t-\tau)^{-1}[y(t)-y(\tau)] \cdot E[y(t)-y(\tau), t-\tau] d \tau, \\
y(t)=\int_{0}^{t}[v(\tau)-\varphi(\tau)] d \tau .
\end{gathered}
$$

Let us introduce the following notation:

$$
T=\sqrt{t}, \quad \Phi(T)=\varphi\left(T^{2}\right), \quad \nabla(T)=v\left(T^{2}\right), \quad Y(T)=y\left(T^{2}\right)
$$

According to our assumptions, Taylor's expansions

$$
\begin{aligned}
& f(x)=\sum_{n=1}^{\infty} f_{n} x^{n} / n ! \\
& \Phi(T)=\sum_{n=0}^{\infty} \Phi_{n} T^{n} / n !
\end{aligned}
$$

have convergence radii equal to $+\infty$ and $T_{0} \in\left(0 ; \sqrt{t_{0}}\right]$, respectively.

Now, perform the following change of variables in (2.2), (2.3)

$$
s=\tau / T^{2}, \quad \lambda=-\xi / 2 T .
$$


We have

$$
\begin{aligned}
& V(T)=J(Y(T), T)-I(T / V, Y), \\
& Y(T)=T^{2} \int_{0}^{1}[V(T \sqrt{s})-\Phi(T \sqrt{s})] d s,
\end{aligned}
$$

where

$$
\begin{gathered}
J=2 \pi^{-\frac{1}{2}} \int_{0}^{+\infty} f^{\prime}(-2 \lambda T) \exp \left[-\frac{Y^{2}(T)}{4 T^{2}}-\frac{Y(T)}{T} \lambda-\lambda^{2}\right] d \lambda, \\
I=(4 \pi)^{-\frac{1}{2}} \int_{0}^{1} V(T \sqrt{s}) \frac{Y(T)-Y(T \sqrt{s})}{T(1-s)^{3 / 2}} \exp \left[-\frac{[Y(T)-Y(T \sqrt{s})]^{2}}{4 T^{2}(1-s)}\right] d s .
\end{gathered}
$$

Introduce the complex variable

$$
T=\varrho e^{i \varphi}
$$

and let

$$
C_{R}=\{T:|T|<R\}
$$

Denote by $\mathcal{H}(R, N)$ the set of functions $\omega(T)$ which are continuous in $\bar{C}_{R}$, analytic in $C_{R}$, and such that

$$
|\omega(T)| \leqslant N, \quad T \in C_{R} .
$$

Given any two functions $\omega_{1}, \omega_{2} \in \mathcal{M}(R, N)$ define the distance

$$
d\left(\omega_{1}, \omega_{2}\right)=\sup _{\sigma_{R}}\left|\omega_{1}(T)-\omega_{2}(T)\right|
$$

Set $R<T_{0}$, define $\Phi(T)$ in $\bar{C}_{R}$ according to (2.5) and choose $N \geqslant \sup _{C_{R}}|\Phi|$. Then, for any $V \in \mathcal{M}(R, N)$, perform the integral in (2.7) defining $Y(T)$, which will belong to $\mathcal{M}\left(R, 2 R^{2} N\right)$.

Next, define $f^{\prime}$ in the whole complex plane according to (2.4) and perform the integrals $J$ and $I$ with respect to the real variables $\lambda$ and $s$. These integrals have no singularities as functions of $T \in C_{R}$ and the singularity w.r.t. $s$ in (2.9) is integrable (because of $(2.7)$ ).

Thus, we have defined a mapping $\hat{V}=\mathscr{C V}=J-I$ of $\mathcal{M}(R, N)$ into a set of functions which are analytic in $C_{R}$ and continuous in $\bar{C}_{R}$.

We shall prove that for suitable $R$ and $N$

(A) $\mathscr{C}$ maps $\mathcal{H}(R, N)$ into itself.

(B) $\mathcal{G}$ is a contraction w.r.t. the metrics $(2.10)$. 
Proof of (A). - The following estimates are easily established

$$
\begin{aligned}
& |Y(T) / T| \leqslant 2 N R, \quad T \in O_{R}, \\
& \left|\frac{Y(T)-Y(T \sqrt{s})}{T(1-s)}\right| \leqslant 2 N R, \quad T \in C_{R} .
\end{aligned}
$$

Recall $\left|f^{\prime}\right| \leqslant M e^{\alpha x^{2}}$ and choose $R<(4 \alpha)^{-\frac{1}{2}}$ so that

$$
\alpha_{0}=1-4 \alpha R^{2}>0
$$

We have, for $T \in C_{R}$

$$
\begin{aligned}
& |J| \leqslant 2 M \pi^{-\frac{1}{2}} e^{N^{2} R^{2}} \int_{0}^{+\infty} \exp \left[-\alpha_{0} \lambda^{2}+2 N R \lambda\right] d \lambda \leqslant 2 M \alpha_{0}^{-\frac{1}{2}} \exp \left[N^{2} R^{2}\left(1+\alpha_{0}^{-1}\right)\right], \\
& |I| \leqslant 2 N^{2} R \pi^{-\frac{1}{2}} e^{N^{2} R^{2}} .
\end{aligned}
$$

Thus, if we fix $N>\max (4 M$, sup $|\Phi|)=\bar{N}$ arbitrarily, an $R_{0}$ can be found depending on $N, \alpha, T_{0}$ such that if $R \leqslant R_{0}$ then $V \in \mathcal{M}(R, N) \Rightarrow|\hat{V}| \leqslant N$, thus concluding the proof of $(\mathrm{A})$.

Proof of (B). - Let $V_{i} \in \mathcal{M}(R, N), i=1,2$ and $Y_{i}$ be defined through $V_{i}$ by (2.7). Then, the following estimates are valid in $C_{R}$

$$
\begin{aligned}
& \delta(Y / T) \leqslant R d\left(V_{1}, V_{2}\right)\left(^{(3)}\right. \\
& \delta\left(\frac{Y(T)-Y(T \sqrt{s})}{T(1-s)}\right)=\left|\frac{T}{1-s} \int_{s}^{1}\left[V_{1}(T \sqrt{\tau})-V_{2}(T \sqrt{\tau})\right] d \tau\right| \leqslant R d\left(V_{1}, V_{2}\right) .
\end{aligned}
$$

After simple calculations one obtains

$$
\delta I \leqslant K(R, N) d\left(V_{1}, V_{2}\right)
$$

where $K$ is a given continuous increasing function of its arguments such that $K(0, N)=0$.

Similarly,

$$
\delta J \leqslant d\left(V_{1}, \nabla_{2}\right) 2 \pi^{-\frac{1}{2}} \int_{0}^{+\infty}\left|f^{\prime}(-2 \lambda T)\right|\left(4 N R^{2}+R \lambda\right) \exp \left[N^{2} R^{2}+2 N R \lambda-\lambda^{2}\right] d \lambda
$$

$\left(^{3}\right) \delta(F(Y, V)) \equiv\left|F\left(Y_{1}, V_{1}\right)-F\left(Y_{2}, \nabla_{2}\right)\right|$ for any function $F$ defined in terms of $V$ and $F$. 
and finally

$$
d\left(\hat{V}_{1}, \hat{V}_{2}\right) \leqslant K_{1}(R, N) d\left(V_{1}, V_{2}\right)
$$

where $K_{1}$ has the properties just described for $K$. This completes the proof of (B).

At this point Banach's Theorem yields the existence of a unique fixed point $\vec{V}$ for $\mathfrak{C}$, belonging to $\mathcal{M}(R, N)$, for any $N>\bar{N}$ and $R$ sufficiently small. This means that the restriction of $\bar{V}$ to the real axis is an analytic function in $[0, R]$ which solves $(2.6)-(2.7)$.

Therefore the problem (1.1)-(1.5) has a solution with $y(t)$ analytic w.r.t. $\sqrt{t}$ in $[0, \sqrt{R}]$. Since it is known that (1.1)-(1.5) cannot have two solutions, the proof of Theorem 1 is concluded, once the results of [1] or standard prolongation are used to continue the domain of analyticity till $t=t_{1}$.

REMARK 2. - A crucial point in the proof of Theorem 1 is the analytic extension to the complex plane of the function

$$
\int_{-\infty}^{0} f^{\prime}(\xi) E\left(Y(T)-\xi, T^{2}\right) d \xi .
$$

Since by definition $\int_{-\infty}^{0}=\lim _{k \rightarrow+\infty} \int_{-k}^{0}$, after the transformation $\lambda=-\xi / 2 T$ this limit becomes $\lim _{k \rightarrow+\infty} \int_{0}^{k / 2 T}$, which is not suitable for the analytic extension, because the variable $\lambda$ in (2.8) would be complex, and convergence of the integral would be ensured only for $\operatorname{Re} T>\operatorname{Im} T$. However, for $T$ real and positive we can say that

$$
J=2 \pi^{-\frac{1}{2}} \lim _{k \rightarrow+\infty} \int_{0}^{k} f^{\prime}(-2 \lambda T) \exp \left[-\frac{Y^{2}(T)}{T^{2}}-\frac{Y(T)}{T} \lambda-\lambda^{2}\right] d \lambda
$$

and it is this form of the integral which has been extended to the complex plane.

\section{3. - Power series expansion of $Y(T)$.}

In this section we derive some recursive relations determining the coefficients in the power expansion of $Y(T)$ :

$$
Y(T)=\sum_{n=1}^{\infty} \frac{Y_{n}}{n !} T^{n}
$$

in terms of the coefficients of the expansions (2.4), (2.5). We shall look for the power series expansions

$$
I=\sum_{n=-1}^{\infty} I_{n} T^{n}, \quad J=\sum_{n=0}^{\infty} J_{n} T^{n}
$$


then, recalling equations (2.6)-(2.7) giving

$$
\frac{1}{2 T} \dot{Y}(T)+\Phi(T)=J-I
$$

we shall impose that the corresponding coefficients in the expansions of the left and of the right hand side of (3.3) coincide.

Let us begin with the term $I$. Setting

$$
z=[Y(T)-Y(T \sqrt{s})] /\left[2 T(1-s)^{\frac{1}{2}}\right]
$$

it is not difficult to obtain

$$
z e^{-z^{2}}=\sum_{n=0}^{\infty} \frac{(-1)^{n}}{n} 2^{-2 n-1}(1-s)^{-n-\frac{1}{2}} \cdot \sum_{r=0}^{\infty} T^{r} \sum_{|j|=r+2 n+1} \prod_{k=1}^{2 n+1} \frac{1-s^{j_{k} / 2}}{j_{k} !} Y_{j_{k}},
$$

where

$$
|j|=\sum_{k=1}^{2 n+1} j_{k}
$$

Now, taking into account that

$$
V(T)=\frac{1}{2 T} \dot{Y}(T)+\Phi(T)
$$

we can insert the expansion

$$
V(T)=\frac{1}{2} Y_{1} T^{-1}+\sum_{n=1}^{\infty}\left(\frac{1}{2 n} Y_{n+1}+\Phi_{n-1}\right) T^{n-1} /(n-1) !
$$

into the integral defining $I$

to get

$$
I=\frac{1}{\sqrt{\pi}} \int_{0}^{1} V(T \sqrt{\delta})(1-s)^{-1} z e^{-z^{2}} d s
$$

$$
\begin{aligned}
I=\sum_{\substack{l=0 \\
l_{2}+l_{2}=l}}^{\infty}(-1)^{l_{1}} \frac{1}{l_{1} ! l_{2} !} 2^{-2 l_{1}-1}\left[\frac{1}{2\left(l_{1}+1\right)} Y_{l_{2}+2}+\Phi_{l_{2}}\right] \\
\cdot \sum_{r=0}^{\infty} T^{l_{2}+r} \sum_{|j|=r+2 l_{1}+1} \mu_{l_{1}, l_{2},\{j\}}^{2 l_{2}+1} \prod_{k=1} Y_{j_{k}} / j_{k} !+ \\
+Y_{1} \sum_{n=0}^{\infty} \frac{(-1)^{n}}{n !} 4^{-n-1} \sum_{r=0}^{\infty} T^{r-1} \sum_{|j|=r+2 n+1} \mu_{n,-1,\{j\}} \prod_{k=1}^{2 n+1} Y_{j_{k}} \mid j_{k} !
\end{aligned}
$$

where $\{j\}$ is the set of the indexes $j_{k}$ appearing in the products and

$$
\mu_{p_{1}, p_{2},\{j}=\frac{1}{\sqrt{\pi}} \int_{0}^{1} s^{p_{2} / 2}(1-s)^{-p_{1}-\frac{2}{2}} \prod_{k=1}^{2 v_{2}+1}\left(1-s^{j_{k} / 2}\right) d s .
$$


To calculate the expansion of $J$, first we write

$$
J=\frac{2}{\sqrt{\pi}} \int_{Y(T) / 2 T}^{+\infty} f^{\prime}(Y(T)-2 T \eta) e^{-\eta^{2}} d \eta,
$$

then we use the expansion

$$
f^{\prime}(X(T)-2 T \eta)=\sum_{n=0}^{\infty} \frac{(2 T)^{n}}{n !} f_{n+1} \sum_{m=0}^{n}(-1)^{m}\left(\begin{array}{l}
n \\
m
\end{array}\right)\left(\frac{Y(T)}{2 T}\right)^{n-m} \eta^{m}
$$

and we define the functions

$$
\psi_{n, m}(\omega)=\frac{2}{\sqrt{\pi}} \omega^{n-m} \int_{\omega}^{+\infty} \eta^{m} e^{-\eta^{2}} d \eta .
$$

Next we consider the expansions

$$
\psi_{n, m}(\omega)=\sum_{l=0}^{+\infty} c_{n, m, l} \frac{\omega^{l} !}{l !},
$$

where it is

$$
c_{n, m, l}=0 \quad \text { for } n>m+l
$$

inserting $\omega=Y(T) / 2 T$ in (3.13) and using (3.11) in (3.10) we get the desired result

$$
\begin{aligned}
& J=\sum_{n=0}^{\infty} f_{n+1} \frac{1}{n !}\left\{(-1)^{n} e_{n, n, 0}(2 T)^{n}+\right. \\
& \left.\quad+\sum_{m=0}^{n}(-1)^{m}\left(\begin{array}{l}
n \\
m
\end{array}\right) \sum_{l=1}^{\infty} c_{n, m, l} \frac{2^{n-1}}{l !} \sum_{r=0}^{\infty} T^{r+n} \sum_{|j|=l+r} \prod_{k=1}^{l} Y_{j_{k}} \mid j_{k} !\right\} .
\end{aligned}
$$

A great simplification of (3.9) and (3.15) is implied by

$$
Y_{1}=0
$$

which is an obvious consequence of the assumption $f(0)=0$.

Indeed, as a result of $(3.16)$, it can be proved that the relations

$$
\left(\frac{1}{2(N+1)} Y_{N+2}+\Phi_{N}\right) / N !=J_{N}-I_{N}, \quad N=0,1, \ldots,
$$

deduced by (3.2), (3.3), are recursive relations for the coefficients $Y_{n}$. As a matter of fact let us show that $I_{N}$ and $J_{N}$ are finite sums containing $Y_{n}$ with $n \leqslant N+1$.

Consider (3.9) with $Y_{1}=0$ and take $l_{2}+r=N>0$, i.e. $r=N-l_{2}$ and $l_{1}=l-l_{2}$, for any $l=0,1, \ldots$, with $l_{2}=0,1, \ldots, \min (l, N-1)\left(l_{2}=N\right.$, and in particular $N=0$, implies $r=0$, i.e. $\{j\}=\{1,1, \ldots, 1\}$ and gives no contribution). The value of $|j|$ in the summation is $N+1+2 l-3 l_{2}$. If $\{j\}$ would contain an index $N_{0} \geqslant N+2$ (say

20 - Annali di Matematica 
the last one) and $j_{k}>1$ for all $k$, then we would have

$$
|j|=N+1+2 l-3 l_{2}=N_{0}+\sum_{k=1}^{2 l_{1}} j_{k} \geqslant N_{0}+4 l_{1} \geqslant N+2+4 l-4 l_{2},
$$

i.e. $0 \geqslant 1+2 l-l_{2} \geqslant 1+l$, which is contradicted by $l \geqslant 0$.

On the other hand for any set $\{j\}$ of $2 l_{1}+1$ elements greater than 1

$$
\sum_{k=1}^{2 l_{1}+1} j_{k} \geqslant 4\left(l-l_{2}\right)+2
$$

i.e.

$$
N+1+2 l-3 l_{2} \geqslant 4\left(l-l_{2}\right)+2,
$$

whence

$$
N \geqslant 2 l-l_{\mathrm{a}}+1 \geqslant 2 l+1
$$

implying that $l$ can take only values not exceeding $[(N-1) / 2]$. Thus we conclude that

$$
\begin{aligned}
& I_{0}=0 \\
& I_{N}=\sum_{l=0}^{[(N-1) / 2]} \sum_{l_{\mathrm{s}}=0}^{l} \frac{(-1)^{l-l_{2}}}{\left(l-l_{2}\right) ! l_{2} !} 2^{-2\left(l-l_{2}\right)-1}\left[\frac{1}{2\left(l_{2}+1\right)} Y_{l_{2}+2}+\Phi_{l_{3}}\right] \\
& \cdot \sum_{|j|=N+1+2 l-3 l_{2}} \mu_{l-l_{2}, l_{2},\{j\}} \prod_{k=1}^{2\left(l-l_{2}\right)+1} Y_{j_{k}} / j_{k} !, \quad N=1,2, \ldots
\end{aligned}
$$

Let us now calculate $J_{N}$ from (3.15). Once again, taking into account $\bar{X}_{1}=0$, we want to show that no index exceeding $N+1$ can be contained in $\{j\}$. Setting $r+n=N$, for any $n=0,1, \ldots, N-1$ and assuming $N_{0} \in\{j\}$ such that $N_{0} \geqslant N+2$ and $j_{k}>1$, we have

$$
|j|=l+N-n \geqslant N_{0}+2(l-1) \geqslant N+2 l,
$$

from which $0>l+n$, contradicting $l \geqslant 1$ in (3.15).

Moreover for any set $\{j\} \notin 1$ of $l$ indexes such that $|j|=l+N-n$

$$
l+N-n \geqslant 2 l,
$$

i.e. $l \leqslant N-n$.

Hence

$$
\begin{aligned}
J_{0} & =f_{1} c_{0,0,0}=f_{1} \\
J_{N} & =(-1)^{N} f_{N+1} \frac{2^{N}}{N !} c_{N, N, 0}+ \\
& +\sum_{n=0}^{N-1} f_{n+1} \frac{1}{n !} \sum_{m=0}^{n}(-1)^{m}\left(\begin{array}{l}
n \\
m
\end{array}\right) \sum_{l=1}^{N-n} e_{n, m, l} \frac{2^{n-1}}{l !} \sum_{[j]=N+l-n} \prod_{k=1}^{l} Y_{j_{k}} / j_{k} ! \quad N=1,2, \ldots
\end{aligned}
$$


Thus, all the coefficients $Y_{n}$ can be calculated from (3.16)-(3.21), recursively.

For instance

$$
\begin{aligned}
& Y_{2}=2\left(f_{1}-\Phi_{0}\right) \\
& Y_{3}=-\frac{8}{\sqrt{\pi}}\left(f_{1}^{2}+f_{2}\right)+\frac{8}{\sqrt{\pi}} f_{1} \Phi_{0}-4 \Phi_{1} \\
& Y_{4}=12\left(f_{3}+f_{1} f_{2}\right)-6 \Phi_{2}-12 f_{2} \Phi_{0}+Y_{3}\left\{\frac{2}{\sqrt{\pi}} f_{1}-\mu_{0,0,\{3\}}\left(Y_{2}+2 \Phi_{0}\right)\right\}
\end{aligned}
$$

with

$$
\mu_{0,0,\{3\}}=\frac{10}{3 \sqrt{\pi}}+\frac{\sqrt{\pi}}{4}
$$

Concerning the coefficients $e_{n, m, l}$ appearing in (3.21) it can be proved that

$$
\begin{array}{ll}
e_{n, m, n-m}=(n-m) ! \Gamma\left(\frac{m+1}{2}\right) / \sqrt{\pi}, & m=0,1, \ldots, n, \\
c_{n, m, l}=\frac{2}{\sqrt{\pi}} \frac{l !}{l-n+m} a_{l-n-1}, & l \geqslant n+1 .
\end{array}
$$

with

$$
a_{j}=\left\{\begin{array}{cl}
0, & \text { if } j \text { is odd } \\
(-1)^{p+1} / p !, & \text { if } j=2 p
\end{array}\right.
$$

$\boldsymbol{c}_{n, m, l}=0$ for $l<n+1, l \neq n-m$.

REMARK 3. - The conditions

$$
Y_{2 n+1}=0, \quad n=0,1, \ldots
$$

are necessary and sufficient (together with the assumptions made on $f$ and $\varphi$ ) in order that $y(t)$ is analytic at $t=0$. These conditions are obviously identical with the recursive relations (1.9).

\section{4. - Proof of theorem 2.}

If $f_{0} \neq 0$ equation (2.2) is replaced by

$$
\begin{aligned}
v(t)=-(\pi t)^{-\frac{1}{2}} f_{0} \exp \left[-y^{2}(t) / 4 t\right]+2 \int_{-\infty}^{0} f^{\prime}(\xi) E(y(t)-\xi, t) d \xi- \\
\quad-\int_{0}^{t} v(\tau)(t-\tau)^{-1}[y(t)-y(\tau)] E[y(t)-y(\tau), t-\tau] d \tau .
\end{aligned}
$$


Hence, after setting

$$
T=\sqrt{t}, \quad \Psi(T)=T \varphi\left(T^{2}\right), \quad W(T)=T v\left(T^{2}\right), \quad Y(T)=y\left(T^{2}\right)
$$

instead of $(2.6),(2.7)$ we get

$$
\begin{aligned}
& W(T)=\bar{J}(Y(T), T)-\bar{I}(T / W, Y)-\pi^{-\frac{1}{2}} f_{0} \exp \left[-\left(\frac{Y(T)}{2 T}\right)^{2}\right], \\
& Y(T)=T \int_{0}^{1} s^{-\frac{1}{2}}[W(T \sqrt{s})-\Psi(T \sqrt{s})] d s,
\end{aligned}
$$

where

$$
\begin{aligned}
& \bar{J}=2 T \pi^{-\frac{1}{2}} \int_{0}^{+\infty} f^{\prime}(-2 \lambda T) \exp \left[-\frac{Y^{2}(T)}{4 T^{2}}-\frac{Y(T)}{T} \lambda-\lambda^{2}\right] d \lambda, \\
& \bar{I}=(4 \pi)^{-\frac{1}{2}} \int_{0}^{1} s^{-\frac{1}{2}} W(T \sqrt{s}) \frac{Y(T)-Y(T \sqrt{s})}{T(1-s)^{\frac{3}{2}}} \exp \left[-\frac{(Y(T)-Y(T \sqrt{s}))^{2}}{4 T^{2}(1-s)}\right] d s .
\end{aligned}
$$

As in Section 2, we can define a mapping $\hat{W}=\overline{\bar{\sigma}} W$ of $\mathcal{H}(R, N)$ into a set of functions which are analytical in $C_{R}$ and continuous in $\bar{C}_{R}$, provided $R<T_{0}$.

Following the same steps as in Section 2 (proof of $(A)$ ) with the appropriate changes, we find that if

$$
W \in M(R, N), \quad \sup _{O_{R}}|\Psi| \leqslant N_{0}
$$

then

$$
\begin{aligned}
& |Y(T) / T| \leqslant 2\left(N+N_{0}\right), \quad T \in O_{R}, \\
& \left|\frac{Y(T)-Y(T \sqrt{s})}{T(1-s)}\right| \leqslant 2\left(N+N_{0}\right), \quad T \in C_{R} .
\end{aligned}
$$

Consequently, for $T \in C_{R}$

$$
\begin{aligned}
|\hat{W}| \leqslant \pi^{-\frac{1}{2}}\left|f_{0}\right| \exp \left[\left(N+N_{0}\right)^{2}\right]+ \\
\quad+2 \alpha_{0}^{-\frac{1}{2}} M R \exp \left[\left(N+N_{0}\right)^{2}\left(1+\alpha_{0}^{-1}\right)\right]+\pi^{\frac{1}{2}} N\left(N+N_{0}\right) \exp \left(N+N_{0}\right)^{2}
\end{aligned}
$$

Similarly, (2.13) and (2.14) of Section 2 are now replaced by

$$
\begin{aligned}
& \delta(Y / T) \leqslant 2 d\left(W_{1}, W_{2}\right) \\
& \delta\left(\frac{Y(T)-Y(T \sqrt{s})}{T(1-s)}\right) \leqslant 2 d\left(W_{1}, W_{2}\right)
\end{aligned}
$$

for any $T \in C_{R} ; W_{1}, W_{2} \in \mathcal{M}(R, N)$. 
Hence

$$
\begin{aligned}
& d\left(\hat{W}_{1}, \hat{W}_{2}\right) \leqslant\left\{\left[2 \pi^{-\frac{1}{2}}\left|f_{0}\right|\left(N+N_{0}\right)+\right.\right. \\
+ & \left.\left.\pi^{\frac{1}{2}}\left(N+\left(N+N_{0}\right)+N\left(N+N_{0}\right)\right)\right] \exp \left[\left(N+N_{0}\right)^{2}\right]+R M F\left(N, N_{0}\right)\right\} d\left(W_{1}, W_{2}\right)
\end{aligned}
$$

where $F\left(N, N_{0}\right)$ is a bounded function of $N$ and $N_{0}$, also depending on $\alpha_{0}$.

Using (4.7) and (4.10) it is easily found that $\overline{\mathscr{C}}$ is a contractive mapping of $\mathcal{M}(N, R)$ into itself for sufficiently small $R$, provided that

$$
\left|f_{0}\right| \leqslant f_{0}^{*}, \quad N_{0} \leqslant N_{0}^{*},
$$

where $f_{0}^{*}, N_{0}^{*}$ are chosen in such a way that the following inequalities are simultaneously solved for some $N>0$ :

$$
\begin{aligned}
& \left\{\pi^{-\frac{1}{2}} f_{0}^{*}+\pi^{\frac{1}{2}} N\left(N+N_{0}^{*}\right)\right\} \exp \left[\left(N+N_{0}^{*}\right)^{2}\right]<N, \\
& \left\{2 \pi^{-\frac{1}{2}} f_{0}^{*}\left(N+N_{0}^{*}\right)+\pi^{\frac{1}{2}}\left[N+\left(N+N_{0}^{*}\right)+N\left(N+N_{0}^{*}\right)\right]\right\} \exp \left[\left(N+N_{0}^{*}\right)^{2}\right]<1 .
\end{aligned}
$$

This concludes the proof of Theorem 2.

As we pointed out in Sec. 1 , the case $f_{0}>0$ in particularly delicate. We recall also that the nonexistence of solutions has been estabilished for some special cases of the above type (see [5]); in the next section we shall point out that from a general point of view a bound on $f_{0}$ is a necessary condition for existence.

Now we want to reconsider the question of uniqueness without any restriction on the class of solutions: from this point of view the following result is a partial extension of Theorem 2.

THEOREM 3. - Assume $\varphi=0$ and $f(x) \leqslant 1(f \not \equiv 1),-\infty<x \leqslant 0$. Then problem (1.1)(1.5) has at most one solution.

Proof. - Let $\left(t^{*}, y, u\right)$ be any solution of (1.1)-(1.5). The function

$$
z(x, t)=-\int_{x}^{y(t)} \int_{\xi}^{y(t)}[u(\eta, t)-1] d \xi d \eta, \quad x<y(t), \quad t \in\left(0, t^{*}\right)
$$

is a solution of

$$
\begin{aligned}
& z_{x x}-z_{t}=1, \quad x<y(t), t \in\left(0, t^{*}\right), \\
& y(0)=0, \\
& z(y(t), t)=z_{\dot{x}}(y(t), t)=0, \quad t \in\left(0, t^{*}\right), \\
& z(x, 0)=-\int_{x}^{0} \int_{\xi}^{0}(f(\eta)-1) d \xi d \eta \equiv g(x), \quad x<0,
\end{aligned}
$$


often referred to as the oxygen diffusion-consumption problem. Recalling Theorem 3.1 of [5] (and extending it to the case of unbounded domains), we have uniqueness if $g \geqslant 0, g^{\prime} \leqslant 0 \quad(g \neq 0)$. Such conditions are actually implied by $f(x) \leqslant 1 \quad(f \neq 1)$ and the theorem is proved.

To conclude this section we remark that all the analysis performed so far can be extended to the two-phase problem. In particular, the extension of Theorem 2 points out that some two-phase problems with initial data jumping at $x=y(0)$ are solvable under no sign restriction and with a proper bound on $|f(0+)-f(0-)|$.

\section{5. - A nonexistence theorem for problem (1.1)-(1.5).}

For the sake of simplicity we assume in the following $\lim _{t \rightarrow 0^{+}} t^{\frac{1}{2}} \varphi(t)=0$.

THEOREM 4. - Under the above assumption, if $f_{0} \geqslant 1$ there is no solution of (1.1)-(1.5) such that $y(t) / t^{\frac{1}{2}}$ has a bounded limit for $t \rightarrow 0^{+}$.

Proof. - Assume (1.1)-(1.5) has a solution such that

$$
\lim _{T \rightarrow 0^{+}} Y(T) / T=\dot{Y}(0) \equiv Y_{1}
$$

exists. Then from (4.2), (4.3)

$$
Y_{1}=2 W(0)=2 W_{0}
$$

and

$$
\begin{aligned}
& W_{0}=-\pi^{-\frac{1}{2}} f_{0} \exp \left[-W_{0}^{2}\right]- \\
& \quad-\pi^{-\frac{1}{2}} W_{0}^{2} \int_{0}^{1}\left[s^{\frac{1}{2}}(1-s)^{\frac{1}{2}}\left(1+s^{\frac{1}{2}}\right)\right]^{-1} \exp \left[-W_{0}^{2}\left(1-s^{\frac{1}{2}}\right)\left(1+s^{\frac{1}{2}}\right)^{-1}\right] d s .
\end{aligned}
$$

It is not difficult to see that the second term is equal to $-W_{0}$ erf $W_{0}$ : Hence (5.2) can be rewritten

$$
f_{0}=-\pi^{\frac{1}{2}} W_{0} \exp \left(W_{0}^{2}\right)\left\{1+\operatorname{erf} W_{0}\right\} .
$$

It can be shown that the right hand side of (5.3) is a decreasing function of $W_{0}$ such that

$$
\lim _{W_{0} \rightarrow+\infty}=1, \lim _{W_{0} \rightarrow+\infty}=-\infty .
$$

Hence the proof of Theorem 4. 
REMARK 4. - For $f_{0}<1$ equation (5.3) provides a $1-1$ correspondence between $f_{0}$ and $W_{0}$, thus determining $\dot{Y}(0)$ in terms of $f_{0}$.

\section{6. - The case of hounded domains.}

In this section we shall study Stefan problems in bounded domains. For sake of brevity, we consider the simple one-phase problem without ablation

$$
\begin{array}{ll}
u_{x x}-u_{t}=0, & -l<x<y(t), t>0, \\
u(x, 0)=f(x), & -l<x<0, \\
u(-l, t)=g(t), & t>0, \\
u(y(t), t)=0, & t>0, \\
\dot{y}(t)=u_{x}(y(t), t), & t>0, \\
y(0)=0, &
\end{array}
$$

where $l \geqslant 0$ is a given constant and $f$ and $g$ are prescribed functions (obviously condition (6.2) is given only when $l>0$ ). Using the terminology of [2] we shall call (6.1)-(6.6) a "Dirichlet-Stefan problem " whereas we shall refer to (1.1)-(1.5) as to a "Cauchy-Stefan problem ». "Neumann-Stefan problems " (i.e. with $u_{x}$ instead of $u$ prescribed on the fixed part of the boundary) could also be considered with obvious changes. The functions $f$ and $g$ are assumed to be continuously differentiable.

The following theorem will be proved

THEOREM 5. - If $g(t)$ is analytic for $t>0$, and $\left|f_{0}\right|$ is less than a suitable $f_{0}^{*}>0$ then problem (6.1)-(6.6) possesses a solution whose free boundary is analytio in $\left(0, t_{1}\right)$.

The assertion of Theorem 5 was already proved in [1] under some additional regularity assumptions on the data (mainly $f(0)=0$ ). We present here a very simple proof of the result.

According to the integral representation techniques and duplicating the arguments of the previous sections (see in particular Section 4) we find in place of $(4.2)-(4.3)$

$$
\begin{gathered}
W(T)=-\pi^{-\frac{1}{2}} f_{0} \exp \left[-\left(\frac{Y(T)}{2 T}\right)^{2}\right]-\pi^{-\frac{1}{3}} f_{0} \exp \left[-\left(\frac{Y(T)+2 l}{2 T}\right)^{2}\right]+ \\
+2 \pi^{-\frac{1}{2}}[f(-l)-g(0)] \exp \left[-\left(\frac{Y(T)+l}{2 T}\right)^{2}\right]+ \\
+\hat{J}(Y(T), T)-\bar{I}(T / W, T)-I_{1}(T / G, Y)+I_{\mathrm{a}}(T / W ; f, Y) \\
Y(T)=T \int_{0}^{1} s^{-\frac{1}{2}} W(T \sqrt{s}) d s
\end{gathered}
$$


where $\bar{I}$ is defined as in Section 4 ,

$$
\hat{J}=2 T \pi^{-\frac{1}{2}} \int_{0}^{1 /(2 T)} f^{\prime}(2 \lambda T) \exp \left[-\frac{Y^{2}(T)}{4 T^{2}}-\frac{Y(T)}{T} \lambda-\lambda^{2}\right] d \lambda
$$

and

$$
\begin{gathered}
I_{1}=2 T^{2} \pi^{-\frac{1}{2}} \int_{0}^{1} G(T \sqrt{s})(1-s)^{-\frac{1}{2}} \exp \left[-\frac{(Y(T)+l)^{2}}{4 T^{2}(1-s)}\right] d s \\
I_{2}=2 T \pi^{-\frac{1}{2}} \int_{0}^{l /(2 T)} f^{\prime}(-2 \lambda T) \exp \left[-\frac{(Y(T)-2 \lambda T+2 l)^{2}}{4 T^{2}}\right]+ \\
+(4 \pi)^{-\frac{1}{2}} \int_{0}^{1} s^{-\frac{1}{2}} W(T \sqrt{s}) \frac{Y(T)+Y(T \sqrt{s})+2 l}{T(1-s)^{\frac{s}{2}}} . \\
\cdot \exp \left[-\frac{(Y(T)+Y(T \sqrt{s})+2 l)^{2}}{4 T^{2}(1-s)}\right] d s .
\end{gathered}
$$

In (6.10) function $G(T)$ is defined to be $\dot{g}\left(T^{2}\right)$ and, by our assumption, is analytic for $T>0$. Consider its domain of analyticity in the plane of the complex variable $T=\varrho e^{i \varphi}$ and let be $D(\varepsilon, R)$ its intersection with the sectorial region defined by

$$
\varepsilon<\varrho<R, \quad|\varphi| \leqslant \pi / 4-\varepsilon,
$$

for given positive $\varepsilon$ and $R$.

Let $T \in D(\varepsilon, R)$ and extend $\hat{J}$ performing the integral along the straight line connecting the origin with $l /(2 T)$. Define the extension of integral in $I_{2}$, similarly. Since $|\arg T| \leqslant \pi / 4-\varepsilon$, all the integrals appearing in the right hand side of (6.7) converge.

Let $\mathcal{H}(\varepsilon, R, N)$ be the set of the functions which are analytic in $D(\varepsilon, R)$, continuous in $\bar{D}(\varepsilon, R)$ and bounded by $N$ : the arguments of Section 4 can be duplicated to show that for $\left|f_{0}\right|$ small enough, $R_{0}$ and $N$ can be found such that (6.7)-(6.8) define a contractive mapping of $M_{(}\left(\varepsilon, R_{0}, N\right)$ into itself, for any $\varepsilon \in\left(0, R_{0}\right)$. This concludes the proof of Theorem 5 .

Clearly, using the sectorial region $D(\varepsilon, R)$ instead of the circle $O \varepsilon$ is a crucial point in the proof; therefore, the same arguments do not apply in proving the analyticity of $Y(T)$ for $T \geqslant 0$.

It must also be noticed that in the present case the integrals containing $f^{\prime}$ in (6.9) and (6.11) are analytic in $D(\varepsilon, R)$ even if $f^{\prime}$ is only continuous. This is easily estabilished remarking that in both integrals $f^{\prime}$ is evaluated on the real axis. Therefore, if we take a sequence $\left\{f^{(n)}\right\}$ of analytic functions converging to $f$ in the $C^{1}$ norm, the corresponding integrals are analytic in $D(\varepsilon, R)$ and continuous in $\bar{D}(\varepsilon, R)$ irrespectively of the analyticity domains of the $f^{(n)}$. Hence it is immediately seen 
that the corresponding sequences of integrals have a uniform limit in $\bar{D}(\varepsilon, R)$, analytic in $D(\varepsilon, R)$.

Addendum.

After this paper was submitted, we have seen the paper The Stefan problem with arbitrary initial and boundary conditions by L. N. TAO, on Quart. Appl. Math., 36 (1978-79), pp. 223-233. There, a two-phase Stefan problem in the semi-infinite region $x>0$ is considered, in the case $s(0)=0$.

A formal expansion of $s(t)$ in a power series of $t^{\frac{1}{2}}$ is found. However, it seems to us that the proof of the convergence in Section 4 is incomplete.

\section{REFERENCES}

[1] A. Friedman, Analyticity of the free boundary for the Stefan problem, Arch. Rat. Mech. Anal., 61 (1976), pp. 97-125.

[2] L. Rubinstein, The Stefan Problem, A.M.S. Translations of Mathematical Monographs, vol. 27, Providence, R.I., 1971.

[3] M. GevRey, Sur les équations aux derivées partielles du type parabolique, J. Math., Ser. 6, 9 (1913), pp. 305-471.

[4] H. GeIman, Analyticity with respect to a parameter of the phase boundary in the solidification of a melt on a plate, in «Papers on the theory of functions and boundary value problems ", Latv. State Univ., Riga (1968), pp. 89-119.

[5] A. Fasano - M. Primickrio, New results on some classical free boundary problems, to appear on Quart. Appl. Math. 